Relations industrielles

Industrial Relations

\title{
La convention collective et les professionnels salariés au Québec
}

\section{Collective Bargaining and the Professional Employee in Quebec}

Jean-Réal Cardin

Volume 21, numéro 2, 1966

URI : https://id.erudit.org/iderudit/027673ar

DOI : https://doi.org/10.7202/027673ar

Aller au sommaire du numéro

Éditeur(s)

Département des relations industrielles de l'Université Laval

ISSN

0034-379X (imprimé)

1703-8138 (numérique)

Découvrir la revue

Citer cet article

Cardin, J.-R. (1966). La convention collective et les professionnels salariés au Québec. Relations industrielles / Industrial Relations, 21(2), 125-142.

https://doi.org/10.7202/027673ar
Résumé de l'article

L'auteur, dans le présent article, tente de faire le point sur un certain nombre d'aspects caractérisant la situation des employés professionnels en ce qui touche leur statut juridique dans le droit québécois du travail, et plus particulièrement en ce qui a trait à l'organisation syndicale et à la négociation collective.
Tous droits réservés @ C Département des relations industrielles de l'Université Laval, 1966
Ce document est protégé par la loi sur le droit d'auteur. L'utilisation des services d'Érudit (y compris la reproduction) est assujettie à sa politique d'utilisation que vous pouvez consulter en ligne.

https://apropos.erudit.org/fr/usagers/politique-dutilisation/ 


\title{
La convention collective et les professionnels salariés au Québec
}

\author{
Jean-Réal Cardin
}

\begin{abstract}
L'auteur, dans le présent article, tente de faire le point sur un certain nombre d'aspects caractérisant la situation des employés professionnels en ce qui touche leur statut juridique dans le droit québecois du travail, et plus particulièrement en ce qui a trait à l'organisation syndicale et d̀ la négociation collective.
\end{abstract}

\section{La situation antérieure au Code du Travail de 1964}

Quand nous parlons, au Québec, des employés professionnels, nous devons établir de claires distinctions entre les différents groupes de personnes à qui cette expression peut s'appliquer. En effet, nulle part en Amérique du Nord autant qu'au Québec, la notion de profession n'a été si hautement caractérisée dans l'esprit populaire aussi bien que dans la réalité du droit.

La notion traditionnelle de "profession " au Québec a été historiquement liée de près à celle de a corporation ", c'est-à-dire un organisme doté par une législation spéciale de la personnalité civile, ayant le pouvoir de représenter et de gouverner ceux qui en sont membres et de contrôler leur conduite professionnelle. La "corporation professionnelle au Québec, dans son sens le plus strict, est un corps quasi-public auquel doivent appartenir les praticiens qui exercent une profession donnée, en règle générale après avoir obtenu un diplôme universitaire, et ceci à l'exclusion de tout autre groupe de personnes. C'est là la forme prédominante d'organisation au Québec pour ce qu'on appelle les \& professions libérales ». Une de ses principales caractéristiques consiste dans les pouvoirs de monopole qu'elle exerce envers ses membres et envers le public en général.

CARDIN, JEAN-RÉAL, avocat (B.C.L., McGill), M.A., Rel. Ind. (Université de Montréal), études graduées en Economique, Sociologie et Relations industrielles (Université de Chicago), directeur du Département des Relations industrielles de l'Université Laval. 
Ainsi, au Québec, nous devons distinguer nettement entre les membres d'une profession libérale érigée en «corporation professionnelle * telle que définie plus haut, et les membres d'autres professions qui ne jouissent pas de ce statut corporatif, ou qui le possèdent à un degré moindre.

Ces derniers, ne sont obligés de devenir et de rester membres d'aucune association pouvant exister dans le champ de leurs activités, même s'ils sont caractérisés par une certaine compétence, généralement acquise au cours d'études spécifiques et reconnues par un diplôme acadérnique.

Au Québec, à l'heure actuelle, nous n'avons pas moins de seize corporations professionnelles dans le plein sens du terme, c'est-à-dire des corporations « fermées. » Certaines d'entre elles représentent ce que l'on pourrait appeler les « vieilles professions libérales comme le Collège des Médecins, le Barreau, la Chambre des Notaires, mais un certain nombre de nouvelles corporations s'y sont ajoutées avec les années : comptables, agronomes, ingénieurs forestiers, etc . . .

Ce qui complique davantage le sujet, c'est le fait qu'un certain nombre de corporations professionnelles, autres que les seize mentionnées plus haut, se sont vu reconnaître certains attributs du statut corporatif dans les années récentes mais ne peuvent pour autant prétendre au titre de « corporations » dans le plein sens du terme. C'est le cas par exemple, de la Corporation des Instituteurs du Québec et de l'Association des Infirmières du Québec. Quant aux instituteurs, ils doivent d'abord faire partie de leur corporation, mais chaque membre est libre, après avoir avisé officiellement la Corporation dans un délai prescrit, de se retirer de ses rangs et d'exercer son activité professionnelle en marge de cette dernière. Cette possibilité de retrait n'existe pas, à notre connaissance, pour les infirmières.

Cependant, dans ces deux cas, les membres sont libres depuis plusieurs années de devenir membres de syndicats d'instituteurs ou d'infirmières, et ceci avec les mêmes droits que ceux de n'importe quelle autre catégorie de travailleurs. A venir jusqu'au présent Code du Travail une telle possibilité n'existait aucunement pour les membres des corporations "fermées ».

Nous avons conservé si vivante au Québec la notion de corporation, et nous avons tenu en si haute estime le prestige qui l'accompagne dans 
notre société, que presque chaque groupe professionnel a tenté de se faire reconnaître par l'Etat un statut corporatif de façon à rehausser la position sociale de ses membres et à sauvegarder leurs intérêts économiques, collectivement et individuellement.

Ainsi au cours des années plusieurs groupes auxquels normalement le statut corporatif n'aurait pas dû être concédé ont de fait reçu au moins un certain nombre d'attributs des vieilles corporations. On peut expliquer en partie une telle situation si l'on se souvient qu'au Québec durant les années de l'entre-deux guerres, à cause de la dépression économique et de la preuve de la faillite de l'organisation libérale de l'économie, un très fort mouvement idéologique s'est développé chez nous qui prétendait réorganiser l'ordre social au moyen d'une certaine structure corporative laquelle devait s'étendre à tout un champ de l'économie ou de l'activité professionnelle. Ce mouvement tirait ses principes et ses postulats de base des encycliques papales, en particulier de l'encyclique Quadragesimo Anno publiée en 1931 et qui préconisait une restauration de l'ordre social selon les lignes d'une structuration corporative des professions et sur l'action autonome de ce qu'on appelait alors les * corps intermédiaires $\$$.

Se fondant sur ce modèle très largement diffusé par certaines élites cléricales et sociales au Canada français, plusieurs groupes professionnels ont eu davantage recours au statut corporatif plutôt que de permettre à leurs membres de s'organiser en associations libres, et ceci, sans tenir compte du fait que dans plusieurs cas, ces derniers auraient eu un plus grand avantage à se grouper en associations de forme syndicale plutôt que corporative, étant donné le statut de salariés de l'immense majorité ou de la totalité de leurs membres.

Comme, d'autre part, notre législation du travail telle qu'édictée en 1944 excluait spécifiquement les membres des corporations professionnelles de sa définition du terme "employé ", il s'ensuivit qu'un grand nombre de salariés professionnels, parce qu'ils étaient membres d'une corporation visée par notre loi des Relations ouvrières se voyaient refuser le droit d'association tel que proclamé et protégé par cette loi et ne pouvaient, en conséquence, participer au processus de la convention collective tel qu'autorisé par cette même loi. Etant des employés professionnels, ils ne pouvaient pas devenir membres d'une association d'employés (un syndicat) au sens de notre loi des Relations ouvrières, et ne pouvaient, en conséquence, forcer la direction des entreprises, l'employeur, à négocier collectivement avec eux selon cette loi. 
Il importe de souligner aussi que, même au sein des plus vieux collèges professionnels, comme le Collège des Médecins, le Barreau, la Corporation des Ingénieurs professionnels du Québec, etc . . ., les conditions d'exercice ont eu tendance à changer rapidement, de sorte qu'une proportion grandissante de leurs membres étaient amenés à exercer leur: profession en tant qu'employés pour les hôpitaux, le gouvernement, les autorités publiques, les entreprises privées, etc.

Le résultat net d'une telle situation fut qu'une proportion toujours croissante d'employés professionnels se trouvaient écartelés entre l'allégeance qu'ils devaient à leur collège professionnel, d'une part, et la nécessité de plus en plus évidente de protéger leur statut économique au moyen de salaires accrus et de meilleures conditions de travail par la voie d'une action syndicale, d'autre part.

Ainsi donc, quel était, essentiellement, la situation des employés professionnels au Québec à venir jusqu'à la promulgation du nouveau Code du Travail en septembre 1964? Elle peut se résumer ainsi : les employés membres d'une des quatorze corporations professionnelles alors visées par la loi des Relations ouvrières de 1944 n'étaient pas considérés au sens de cette dernière loi comme étant des « salariés ». Ils étaient spécifiquement exclus de la définition du terme * salarié * de l'article 2 de la loi.

Est-ce à dire que les membres de ces corporations visées à l'article 2 de cette loi étaient privés de tout droit à l'association syndicale et à la convention collective selon le droit du travail? La réponse est non, du moins en théorie juridique.

Il existe au Québec un texte statutaire datant de 1924: la loi des Syndicats professionnels dont le but général est de permettre, à certaines conditions, aux personnes qui sont membres d'une même profession et qui exercent des emplois connexes ou des métiers similaires, de se constituer en association, en syndicat professionnel.

Cette loi prévoit un mode d'incorporation pour les associations qui se créent sous son empire, par lequel elles acquièrent la personnalité civile ce qui a pour effet de les doter de tous les attributs généralement dévolus aux personnes morales, tout en leur octroyant un certain nombre de pouvoirs spécifiques quant à l'action collective et à la représentation des membres et du groupe lui-même. Les syndicats ainsi incorporés 
sont vraiment des entités juridiques au plein sens du terme. Un de leurs objets principaux était, à venir jusqu'au nouveau Code du Travail, de négocier des conventions collectives et de conclure de telles conventions, lesquelles donnaient ouverture à tous les droits et recours prévus par la loi pour la sanction des obligations (Loi des Syndicats professionnels, S.R.Q. 1941, Ch. 162, art. 24).

Notons que cette loi, bien qu'elle ait été adoptée d'abord au bénéfice des associations de travailleurs industriels, est en fait un texte de portée beaucoup plus générale qui permet aux associations professionnelles d'acquérir la personnalité juridique, qu'il s'agisse d'associations d'employeurs, de travailleurs professionnels, ou d'employés ordinaires, dans l'industrie, le commerce, les services, etc. Il convient de noter enfin (et ce point est très important) qu'il s'agit d'une loi dont l'utilisation n'est que volontaire ou permissive, en ce sens, par exemple, qu'elle n'établit aucune obligation pour quelqu'employeur que ce soit de négocier collectivement avec une association de ses travailleurs ainsi constituée.

Il reste cependant que les employés professionnels, membres d'une corporation fermée, pouvaient s'organiser en vertu de cette loi et tenter de négocier collectivement avec un employeur. Cette interprétation, toutefois, n'a pas été admise sans difficulté de la part des experts juridiques, des pouvoirs publics et surtout des représentants des corporations professionnelles.

Une question, par exemple, était la suivante : Est-ce que ces syndicats, incorporés mais non accrédités en vertu de la loi des Relations ouvrières, avaient le droit de faire la grève pour obtenir par ce moyen la reconnaissance d'un employeur? Une autre question était la suivante : est-ce que les membres d'une corporation professionnelle qui dans sa charte, dans ses règlements ou dans son code d'éthique prohibait l'organisation syndicale de ses membres, avaient le droit de former un groupe incorporé sous l'empire de la loi des Syndicats professionnels, même si cette procédure ne conférait aucun droit spécifique susceptible de forcer l'employeur à négocier collectivement avec lui? Quelle loi l'emportait sur l'autre? Le principe général d'association reconnu par le statut de 1924 ou la prohibition d'adhérer à un syndicat énoncée dans le code d'éthique ou dans les règlements d'une corporation professionnelle?

Nous rappelons ces quelques sujets de controverse parce qu'ils ont déjà fait l'objet de plusieurs discussions légales dans les années qui ont 
précédé l'adoption du nouveau Code du Travail, et à l'occasion des débats qui ont entouré l'adoption du Code.

Cependant le fait demeure, qu'en pratique, il n'y avait pas de conventions collectives dans les années récentes pour les employés professionnels membres des corporations au Québec. Nous devons mentionner toutefois qu'un certain nombre de syndicats professionnels ont été constitué sous l'empire de la loi de 1924 durant ces années récentes, comme ce fut le cas par exemple de certains groupes de spécialistes dans la profession médicale. Mais ces groupes, pour des raisons évidentes, ne négociaient pas collectivement dans le sens des lois du travail; ils n'étaient que des agents de représentation d'intérêts similaires à l'intérieur du corps médical et vis-à-vis les autorités hospitalières.

Telle était la situation pour les membres des corporations fermées à venir jusqu'au nouveau Code du Travail.

Quant aux autres classes d'employés professionnels, nous avons déjà mentionné le cas des infirmières et des instituteurs lesquels, tout en étant organisés en "corporation", avaient le droit de faire partie d'un syndicat et jouissaient exactement du même statut que les autres groupes d'employés quant au droit de négocier collectivement, de s'affilier à une centrale syndicale, de faire la grève, etc. Ils étaient en un mot (et le sont encore) couverts, comme tout autre groupe de salariés, par les lois générales du travail au Québec.

La même situation prévalait évidemment pour les autres groupes d'employés professionnels non compris parmi ceux mentionnés jusqu'ici : les diplômés universitaires en général, non membres d'une corporation visée par la loi des Relations ouvrières, aussi longtemps que ces personnes satisfaisaient à la définition du terme * salarié * dans cette loi.

Il convient de remarquer toutefois qu'aucun de ces groupes de professionnels n'essaya, à venir jusqu'à tout dernièrement, de se constituer en syndicat dans le but de négocier collectivement une convention collective de travail. La plupart d'entre eux, au contraire, ont essayé historiquement de promouvoir leur statut économique et social au moyen d'une certaine forme de corporation professionnelle et de rejoindre si possible le plein statut d'une corporation fermée comme c'était le cas pour les vieilles professions libérales. 


\section{Les développements depuis 1960 et le régime créé par le Code du Travail}

Avec l'élection du gouvernement libéral en 1960, un mouvement vers la révision de ces vieilles positions dans le champ des professions s'est développé graduellement, ce qui a amené la législature à sanctionner d'importants changements relativement au statut des employés professionnels dans la législation ouvrière.

Le Conseil supérieur du Travail avait été ressuscité par le premier ministre Barrette pendant son court règne comme premier ministre d la suite du décès soudain du premier ministre précédent, Paul Sauvé, en 1959, et le premier mandat de ce Conseil avait été de préparer une nouvelle pièce de législation appelée le Code du Travail, entreprise qui avait été abandonnée depuis 1953 lorsque le premier Conseil supérieur du Travail avait cessé ses activités à cause de difficultés rattachées justement à cette question de la refonte des lois du travail. Le premier ministre Duplessis n’avait pas approuvé le projet de loi présenté alors par le Conseil du temps.

Vers le même temps certains événements sont survenus qui révélèrent une certaine maladaptation, un certain malaise dans le monde professionnel au Québec. Des discussions acerbes eurent lieu au sein de la Corporation des Instituteurs du Québec quant à l'accent que cette organisation devait mettre sur l'action corporative d'une part, et l'action syndicale, d'autre part. Autrement dit, quelle importance relative devaiton donner à l'action "professionnelle " et à l'action revendicative, au sein de la Corporation.

Le gouvernement libéral élu en 1960, sous les pressions de certains groupes professionnels, a continué au cours des années, d'accorder des chartes corporatives à certains de ces groupes. Le mouvement organisé du travail devint alors très critique au sujet de ces nouvelles corporations et les centrales syndicales (la Fédération des Travailleurs du Québec et la Confédération des Syndicats nationaux) manifestèrent ouvertement leur désapprobation face à la prolifération de ces soit-disant corporations. Elles attaquaient en même temps l'ostracisme dont étaient l'objet les membres salariés des corporations professionnelles visées par la loi des Relations ouvrières en ce qui a trait au droit d'association et à la convention collective reconnu par cette loi. 
A l'intérieur des vieilles corporations des points d'interrogation se posèrent quant au besoin pour leurs membres salariés de former des syndicats comme salariés en vue de protéger et de promouvoir leurs intérêts économiques sur le marché du travail.

Nous savons tous que le mouvement dans cette direction se développa très fortement surtout au sein de la Corporation des Ingénieurs professionnels du Québec, où une véritable révolution éclata au cours de l'année 1962. Plus de $90 \%$ des membres de cette Corporation étaient de simples salariés alors que la direction était largement dominée par les membres acquis aux idées patronales et recruté à même les bureaux de direction des grandes firmes industrielles ou d'ingénieurs-conseils. Les conditions de travail et d'emploi des ingénieurs salariés étaient généralement pauvres; leur rémunération était faible, comparée à celle d'autres groupes d'employés professionnels ou même (toute proportion gardée) à celles des techniciens ou des ouvriers qualifiés auxquels ils étaient souvent identifiés quant à leurs fonctions réelles et à leur statut.

Une lutte amère se développa entre la direction de la Corporation et les porte-parole de l'action et de l'organisation syndicales à l'intérieur de la Corporation. Le problème atteignit un point culminant pendant les discussions qui entourèrent l'étude du nouveau Bill de la Corporation des Ingénieurs professionnels du Québec et du nouveau Code du Travail, discussions qui eurent lieu à peu près vers le même temps.

Les ingénieurs partisans du syndicalisme, supportés efficacement par la CSN, protestaient contre la teneur de l'article 3.05 du code d'éthique de la Corporation à l'aide duquel on prétendait prohiber l'organisation syndicale des ingénieurs sous le prétexte qu'une telle participation de leur part au mouvement syndical les conduirait vers une philosophie et vers certaines méthodes d'action incompatibles avec un vrai "professionalisme *, comme par exemple, l'usage de la grève et du piquet. Ils amorcèrent une campagne afin que les restrictions que comportait la notion de salarié dans la loi de Relations ouvrières soient abandonnées dans le nouveau Code comme étant de nature discriminatoire en limitant indûment le droit des salariés professionnels de s'organiser et de négocier collectivement selon la loi.

Et sans attendre plus longtemps, c'est-à-dire avant même la sanction du nouveau Code du Travail et le retrait, en conséquence, de l'article 3.05 du code d'éthique de leur Corporation, les ingénieurs salariés com- 
mencèrent, avec l'aide technique de la CSN, d'organiser des syndicats d'ingénieurs salariés en utilisant les possibilités légales offertes par la loi des Syndicats professionnels afin d'acquérir un statut juridique et un caractère représentatif. Ils agirent ainsi d'abord à la Ville de Montréal, puis à la Commission Hydroélectrique de Québec, et peu de temps après vis-d-vis le gouvernement du Québec lui-même.

Ces syndicats ainsi créés sont maintenant tous rattachés à une fédération d'ingénieurs professionnels, laquelle est elle-même affiliée à la CSN, et le mouvement semble devoir se répandre chez tous les effectifs salariés de la profession dans quelqu'entreprise, privée ou publique, qu'ils pratiquent leur art. Sous ces pressions et sous certaines autres émanant des cercles académiques, le gouvernement sanctionna le nouveau Code du Travail qui entra en vigueur en septembre 1964.

Quel est donc, sur le plan juridique, le statut des salariés professionnels sous l'empire des lois actuelles du travail au Québec? Nous pouvons brièvement le résumer ainsi : premièrement, il n'y a plus de restriction dans la définition du mot salarié dans le Code du Travail relativement aux membres des corporations professionnelles quelles qu'elles soient. Du moment qu'un employé professionnel répond aux critères généraux définis dans le Code pour le terme salarié, il se trouve placé sur le même pied que n'importe quel autre employé salarié, et il peut avec d'autres personnes de même condition faire partie d'un syndicat. Ce dernier peut recevoir l'accréditation syndicale, négocier collectivement, faire la grève, etc., sous l'empire des prescriptions générales du Code, comme toute autre association de salariés. La seule restriction que le Code retient en ce qui a trait aux salariés professionnels, c'est-àdire aux membres des corporations visées par son texte, c'est celle selon laquelle les membres de chacune de ces corporations doivent constituer un groupe distinct pour les fins du Code, c'est-à-dire qu'ils doivent former une unité distincte pour les fins de la négociation collective. Cette dernière obligation va un peu plus loin dans la voie de la restriction que ce que la Loi Taft-Hartley, aux Etats-Unis, permet pour des classes semblables d'employés. La loi américaine, pour sa part, laisse aux employés professionnels un certain choix quant à leur inclusion dans une unité de négociation plus large ou, selon leur désir, de ne former qu'une unité bien à eux, à l'exclusion de tout autre groupe.

\section{Le syndicalisme de cadres et le Code du Travail}

Ce qui vient d'être dit ne révèle toutefois qu'une faible partie de ce qui se passe au Québec dans le champ des relations de travail pour les 
employés professionnels. Quand les ingénieurs réussirent l'incorporation de leur syndicat sous la loi des Syndicats professionnels en dépit de l'opposition farouche de leur propre corporation, et lorsqu'ils réussirent à convaincre les pouvoirs publics d'abandonner les restrictions traditionnelles visant les professionnels salariés, ils gagnèrent la bataille du syndicalisme nord-américain, si l'on peut dire, mais ils n'obtinrent pas par le fait même la reconnaissance légale d'une nouvelle variété de syndicalisme chez nous, variété qu'ils avaient défendue depuis le début de leur action et qui est connue sous le nom de " syndicalisme de cadres".

L'expression " cadre » est originaire de France et elle couvre ces groupes d'employés dans l'industrie, le commerce ou dans tout autre secteur, qui ne sont pas de purs exécutants mais qui, à cause d'une com. pétence particulière dans le champ de la technique, de l'administration ou du commerce, ou encore à cause d'un certain degré d'autorité dans le processus de la direction des entreprises, sont situés quelque part entre le conseil de direction et la main-d'oeuvre ordinaire. En Europe et en France plus particulièrement, il existe des syndicats pour ces groupes de salariés que nous appelons les cadres.

Aux Etats-Unis aussi bien qu'au Canada, suivant en cela l'esprit de la loi Taft-Hartley, nous n'acceptons pas ces distinctions à l'intérieur du bloc de la direction des entreprises. Aussitôt qu'un employé assume une certaine participation, fût-elle minime, dans le processus de la direction, il est assimilé d'emblée à la direction de l'entreprise et ne peut profiter des protections de la loi en matière d'organisation, d'accréditation et de convention collective.

Notre Code du Travail au Québec respecte cette notion-bloc de l'autorité dans la direction tout comme notre loi des Relations ouvrières de 1944 le faisait à venir jusqu'à la promulgation du Code. Ainsi donc, lorsque le Code permet aux professionnels salariés de former des syndicats et de négocier collectivement, cette nouvelle latitude ne vaut que pour ceux qui sont de purs exécutants, des salariés ordinaires selon la terminologie nord-américaine du terme.

Le résultat net de cette situation est que, dans le cas des ingénieurs par exemple, et aussi pour ce qui est des autres professions libérales jusqu'à un certain point, une large proportion de ces derniers participent techniquement ou autrement à la direction et sont en conséquence privés du droit d'organisation et d'accréditation. Ainsi, la possibilité de 
former de vastes syndicats forts et efficaces, homogènes professionnellement, est encore très faible pour un grand nombre de professionnels. Les seuls syndicats permis par le Code du Travail sont en quelque sorte vidés de leurs meilleurs éléments, de ceux qui ont le plus d'expérience et qui seraient les plus aptes à faire profiter les éléments plus jeunes de leur compétence professionnelle et de leurs éclats de services, au sein du syndicat, car généralement ils occupent des fonctions de responsabilité dans la hiérarchie. Dans certains cas, la seule force du nombre n'existe même pas pour ceux qui pourraient se qualifier au terme de la loi comme étant de purs salariés.

Enfin, si nous nous rappelons que le Code du Travail, à son article 20 , prescrit que les membres de chacune des corporations professionnelles visées dans son texte doivent former un groupe distinct pour fin d'accréditation, nous pouvons avoir une idée assez précise de l'éparpillement dans lequel ils peuvent se trouver face à la direction et au processus de la convention collective.

Tout ceci a mené à la situation paradoxale suivante : les ingénieurs, qui ont été parmi les premiers à faire la bataille en vue d'obtenir pour les professionnels le droit à l'accréditation sous l'empire du Code du Travail sont maintenant les tout premiers à passer outre à cette loi et à négliger l'utilisation des étapes nécessaires à l'accréditation, tout en développant l'action du syndicalisme de cadres. En d'autres mots, ils ont simplement tendu vers l'obtention d'un statut corporatif pour leurs groupes en les constituant selon la loi des Syndicats professionnels, et ils ont essayé d'amener dans leurs rangs tous les ingénieurs à l'emploi d'un employeur donné, que ces ingénieurs soient ou non membres de la direction au sens du Code du Travail. Le seul critère d'exception qu'ils tentent de faire accepter par leur employeur lors des négociations, est celui qui vise ceux des membres de la profession qui, selon leurs fonctions, ont le pouvoir d'embaucher, de licencier ou de promouvoir d'autres ingénieurs. C'est-à-dire ceux qui, réellement, représentent la direction dans ses relations avec ses employés-ingénieurs. Même si un ingénieur a charge de diriger un personnel non professionnel, à l'occasion de travaux de génie (hommes de métiers, journaliers, ou autres) si cet ingénieur ne dispose pas de l'autorité mentionnée plus haut sur d'autres ingénieurs, il devrait, au dire des ingénieurs syndiqués, faire partie du syndicat de sa profession.

Si des ingénieurs travaillent en équipe avec d'autres membres de leur profession mais ne font qu'assumer une responsabilité scientifique ou 
technique à l'égard du groupe qu'ils dirigent, même s'ils ont, par exemple, des rapports à faire et à signer quant à la qualité du travail etc., ils devraient pouvoir faire partie, à leurs yeux, du syndicat d'ingénieurs pour l'excellente raison que la décision finale ne leur appartient pas en matière de congédiement ou de promotion.

Les moyens utilisés par le syndicalisme de cadres afin d'atteindre ses objectifs sont l'action directe, la discussion directe avec l'employeur et la reconnaissance de facto par ce dernier du groupe impliqué. Evidemment, l'employeur est libre juridiquement de reconnaitre ou de ne pas reconnaître un tel syndicat. Rien, nous le répétons, dans la loi des Syndicats professionnels ne lui impose d'entrer en négociation avec un groupement créé sous l'empire de cette dernière loi.

Si l'employeur accepte le syndicat et le reconnait, il n'y a aucun problème. S'il résiste, l'action directe peut être utilisée et l'on peut avoir recours à l'arme de la grève afin de forcer l'employeur à reconnaître le syndicat (souvent ces grèves sont appelées " journées d'études »). Toute cette action est faite sans avoir aucunement recours aux prescriptions du Code du Travail. A un certain point de vue une telle procédure peut être tenue pour illégale car elle ignore les obligations stipulées par le Code du Travail relativement aux étapes à franchir avant le déclenchement de la grève. Ce dernier établit clairement dans son article 94 qu'il est prohibé de faire la grève aussi longtemps qu'une association d'employés n'a pas été accréditée ou reconnue et n'a pas obtenu le droit à la grève selon les prescriptions du Code. Mais comme nous sommes ici en face d'une association qui n'est pas totalement formée de salariés au sens du Code du Travail, il existe des opinions juridiques à l'effet que le Code ne s'appliquerait pas à de telles associations, lesquelles seraient libres de faire une grève d'organisation si elles croient qu'il est nécessaire pour elles d'agir ainsi.

Quelle que soit la réponse à cette question controversée, c'est exactement le genre d'action que les ingénieurs du Québec ont suivi jusqu'ici. Ils ont ainsi réussi à obtenir la reconnaissance de facto de la Ville de Montréal et de signer une convention collective de travail très avantageuse en mai de l'année dernière, après avoir menacé d'aller en grève. Ils ont usé de la même stratégie vis-à-vis l'Hydro-Québec, à peu près vers le même temps, mais dans ce cas ils durent avoir recours à la grève (aux journées d'études) durant plus d'un mois afin d'en arriver à un règlement avec cet employeur. La raison de cette grève est la difficulté des parties de s'entendre sur ceux, parmi les ingénieurs, qui pourraient 
faire partie de l'unité de négociation, c'est-à-dire jusqu'à quel niveau de responsabilité, le syndicat pouvait prétendre avoir le droit de représenter des ingénieurs collectivement. Sur les cinq cent cinquante-cinq ingénieurs employés à l'Hydro, quatre cent quarante furent finalement reconnus comme susceptibles d'être représentés par le Syndicat des Ingénieurs de l'Hydro-Québec, alors que si l'accréditation selon les prescriptions du Code avait été requise, seulement deux cent quatrevingts d'entre eux auraient pu être couverts par la convention collective.

On peut donc dire que les ingénieurs professionnels, par leur action, sont vraiment en voie de créer du "droit nouveau » dans les relations de travail au Québec. Ils contribuent à ouvrir à travers un processus sociologique de nouvelles dimensions juridiques aux concepts traditionnels des relations industrielles, concepts que nous avons connus jusqu'ici au Québec et même ailleurs en Amérique du Nord. Il est bon de rappeler que la première poussée dans ce sens avait été donnée par la grève des réalisateurs de Radio-Canada à Montréal il y a quelques années mais il faut remarquer qu'il ne s'agissait alors que d'un cas limité et en quelque sorte particulier. Ce qui est nouveau dans le mouvement actuel, c'est qu'il tend à se répandre à l'ensemble des employés professionnels dans la fonction et dans les services publics, et semble devoir éventuellement se propager à l'ensemble des secteurs privés de l'économie. Nous pouvons soupçonner, toutefois, que cette réunion d'employés supérieurs et de simples salariés au sein d'une même association syndicale ne va pas sans remettre en question les structures d'autorité traditionnelles en Amérique du Nord, et pose des problèmes sérieux d'allégeance aux cadres syndiqués d'une entreprise.

\section{Les employés civils professionnels et la loi de la Fonction publique}

Nous devons nous rappeler ici que la nouvelle loi de la Fonction publique du Québec (Bill 55), promulguée en août 1965, reconnaît le droit pour les employés civils de s'organiser en syndicats et de négocier collectivement avec les pouvoirs publics. Cette nouvelle loi, tout en considérant les employés gouvernementaux comme étant couverts d'une façon générale par le Code du Travail, apporte certaines limitations aux droits reconnus généralement par ce Code, limitations qui ne détruisent pas dans l'ensemble la substance des droits reconnus par le Code du Travail. ${ }^{1}$

(1) Pour une analyse plus détaillée de la loi de la Fonction publique (Bill 55), cf. infra: Section "Commentaires", dans ce même numéro. 
Les membres des corporations professionnelles mentionnées au Code du Travail qui sont employés du gouvernement au sens du Code peuvent recevoir une accréditation, soit pour chaque groupe professionnel particulier, ou si une majorité dans chaque groupe le désire, ils peuvent se réunir dans une association d'envergure plus large groupant plusieurs groupes de professionnels afin de négocier collectivement pour tous ces groupes. Ils peuvent même se joindre à d'autres groupes d'employés professionnels qui ne sont pas membres des corporations mentionnées au Code (v.g. économistes, biologistes, etc.) afin de former une association interprofessionnelle qui négocierait avec le gouvernement. Comme nous l'avons déjà noté plus haut, cette possibilité pour les fonctionnaires professionnels de former des unités de négociation interprofessionnelles n'existe pas, si l'on considère le texte lui-même de l'article $20 \mathrm{du}$ Code du Travail, pour les employés professionnels du secteur privé de l'économie.

Mais encore ici le gros problème, semble-t-il, est celui de délimiter les frontières qui doivent exister entre les employés civils professionnels qui sont des salariés au sens du Code et ceux qui n'en sont pas. Lorsqu'on sait que la définition de l'unité d'accréditation, de même que la décision quant à qui est un "salarié », incombent à la Commission de Relations de travail, nous pouvons soupçonner que les critères traditionnels développés par cette Commission dans le secteur privé seront éventuellement déficients quand il s'agira de définir les unités de négociation dans la fonction publique.

Les mêmes attitudes qui ont été adoptées par les ingénieurs de l'Hydro-Québec quant au syndicalisme de cadres auront tendance, croyons-nous, à être utilisées lorsqu'il s'agira de définir les unités de négociation dans la fonction publique.

Dans les emplois gouvernementaux, où l'imbrication des fonctions, des classes et des grades est si grande, il serait dans notre opinion totalement irréaliste d'utiliser les vieux critères de notre droit du travail traditionnel. Le législateur semble d'ailleurs avoir prévu la difficulté, en ce qui a trait aux professionnels, en disant à la section 71 du Bill 55 que l'accréditation sera accordée initialement par le Lieutenantgouverneur en Conseil et "seulement selon la recommandation d'un comité conjoint constitué à cette fin par le Lieutenant-gouverneur en Conseil et dont la moitié des membres seront les représentants du groupe intéressé .. 
Mais même dans les secteurs privés de l'économie où les changements technologiques et l'automatisation changent les structures traditionnelles de l'emploi et provoquent la venue dans l'industrie de nouvelles classes de spécialistes, techniciens et professionnels, travaillant comme salariés à des niveaux intermédiaires de la direction, il est inévitable que le syndicalisme de cadres ait tendance à se répandre jusqu'aux membres de ces nouveaux groupes d'employés professionnels ou semiprofessionnels qui s'accroissent sans cesse. Il faut reconnaître qu'une telle tendance ne va pas sans créer des difficultés sérieuse sur le plan juridique et sociologique, au moins dans un avenir immédiat.

\section{L'organisation syndicale chez les professionnels salariés: un bref aperçu}

Il serait peut-être opportun, en guise de conclusion, de présenter un bref aperçu du mouvement syndical chez les professionnels salariés au Québec face à la convention collective. En plus des enseignants (laïcs aussi bien que religieux) des degrés élémentaire, secondaire et technique dans les institutions privées et publiques, lesquels sont généralement bien organisés et négocient collectivement depuis plusieurs années dans certains cas; en plus des infirmières et des autres catégories professionnelles ou para-professionnelles du monde médical dont les associations négocient avec les associations d'hôpitaux et le Ministère de la Santé, il existe plusieurs autres groupes spécialisés qui se sont formés récemment en associations vouées à la convention collective: par exemple, les inspecteurs d'écoles, les principaux d'écoles, les professeurs des beaux-arts, les professeurs des écoles normales, etc. Nous pouvons remarquer ici que les principaux d'écoles, qui ont récemment signé une convention collective avec la Commission des Ecoles catholiques de Montréal, constituent un autre exemple d'un syndicat de cadres reconnu de facto, et cette fois, par l'autorité scolaire la plus importante au Québec.

Dans le monde médical, il n'existe pas moins de vingt-trois syndicats de différentes catégories spécialisées. La plupart d'entre eux sont incorporés sous la loi des Syndicats professionnels et ont récemment donné naissance à une fédération des syndicats de médecins spécialistes. Tout à côté existe la Fédération des Omnipraticiens du Québec, composée de onze unités régionales. Ces deux fédérations, sauf erreur, ont tout récemment donné naissance à une vaste organisation de médecins dont un des premiers objectifs est de servir d'agent négociateur pour le 
corps médical en vue des négociations avec les pouvoirs publics relativement au système d'assurance-santé universel que le Québec est sur le point d'adopter.

Dans la Fonction publique le mouvement vers l'organisation syndicale des professionnels est tout aussi impressionnant. En plus des ingénieurs qui se totalisent aux alentours de trois cent soixante-quinze membres dans leur groupe, il existe des syndicats d'ingénieurs forestiers, d'arpenteurs-géomètres, ainsi qu'un vaste syndicat interprofessionnel composé de biologistes, chimistes, comptables, géographes, géologues, spécialistes en finance, médecins vétérinaires, économistes, physiciens, diététiciennes, actuaires, etc. Récemment, un syndicat d'avocats a été créé dans la Fonction publique en dépit des objections du Barreau. Les agronomes et les travailleurs sociaux sont en voie de se joindre aux autres si ceci n'est pas déjà fait.

Tous ces groupes font partie depuis le mois de mars de l'an dernier de ce qu'on appelle "le Conseil syndical des professionnels du gouvernement du Québec ». Ce Conseil représente tout près de huit cents membres sur un total d'environ mille six cents individus susceptibles d'être couverts par une convention collective sur un total de deux mille cent employés professionnels dans la Fonction publique.

Le Syndicat des Ingénieurs du Gouvernement du Québec, avec son projet de convention collective, semble en voie d'établir le modèle selon lequel les négociations entre les autorités gouvernementales et les syndicats de professionnels pourraient éventuellement avoir lieu. Ce projet de convention comprend trois sections: a) une section qui s'occupe spécifiquement des ingénieurs eux-mêmes; b) une seconde section touche d'une façon plus générale les intérêts professionnels susceptibles de s'appliquer à tous les employés professionnels dans la Fonction publique; c) enfin, une troisième section de portée encore plus générale s'occuperait des problèmes touchant les conditions de base applicables à l'ensemble de la Fonction publique, aussi bien professionnelle que nonprofessionnelle.

Ainsi, avec le temps, un cartel des syndicats de tous les employés gouvernementaux, professionnels et non professionnels, pourrait être formé en vue de discuter à un certain niveau les questions d'intérêt général à tous, pour ensuite aborder les problèmes plus spécifiques touchant chaque classe d'employés en particulier. 
Il vaut peut-être la peine, en terminant, de rappeler que le premier contrat collectif conclu par les ingénieurs avec la Ville de Montréal a signifié pour ces derniers des gains vraiment impressionnants en matière d'augmentations de salaire, d'heures de travail, de primes de surtemps, de règles de promotion, d'avantages sociaux et de privilèges professionnels. La Fédération des Ingénieurs du Québec (laquelle, sauf erreur, a élargi ses cadres et est devenue tout récemment la Fédération des Ingénieurs et Cadres du Québec) étant affiliée à la Confédération des Syndicats nationaux, il ne serait pas surprenant, avec les nouvelles possibilités offertes par la loi de la Fonction publique, de voir l'ensemble des syndicats professionnels d'employés civils s'affilier à cette centrale syndicale comme les autres fonctionnaires le sont déjà.

Il sera intéressant, à la lumière des événements actuels et de ceux qui se préparent, de surveiller attentivement ce mouvement d'ensemble des salariés professionnels vers l'action syndicale et la convention collective dans notre milieu.

\section{COLLECTIVE BARGAINING AND THE PROFESSIONAL EMPLOYEE IN QUEBEC}

When we speak, in Quebec, of professional employees, we have to distinguish rather sharply between the various groups of persons to which this expression may apply. We have to distinguish between professionals, members of a liberal profession governed by a professional corporation (which we call a a closed corporation $\gg$ ) and those who are not governed by such a corporation.

Up to the enactment of the new Labour Code, in September 1964, employees who were members of one of the fourteen * corporations ", contemplated by the Labour Relations Act of 1944, were not considered to be « employees » under the terms of that Act. They were specifically excluded from the definition of x employee $\otimes$.

Professional employees, members of a closed corporations », could generally organize under the Professional Syndicates' Act and try to negotiate collectively with an employer. However, the fact remains that, in practice, there was no collective bargaining going on up to the recent years for the professional workers, members of corporations, in Quebec.

Other classes of professionals, insofar as they were « employees », had the full right to join a union and were exactly on the same footing as any other group of « employees » as to the right to bargain collectively, affiliate to a central body, go on strike, etc. They were, in a word, and still are, covered, as any other group, by the labour laws of Quebec. It is safe to say, however, that none of these groups of « professionals » even tried, up to now, to form and join unions in order to bargain collectively. 
Starting in the year 1960, a series of events took place which transformed this situation. «Employee» engineers were the first to overthrow the regulations of their "corporation " and to initiate a movement toward what we call in Quebec a «syndicalisme de cadres ». They fought to have professional employees, members of corporations, brought under the definition of "employee " in the Labour Code enacted in 1964, and they succeeded.

So, there is no longer any restriction in the definition of "employee " in the Code, concerning members of professional corporations. As long as they comply with the general criteria defined in the Code for the term "employee ", they are on exactly the same footing as any other kind of " employees " and they may form unions, receive certification and bargain collectively under the general prescriptions of the law.

However, when the Code permits professionals to form unions and bargain collectively, this new liberty is available only to those who are pure "employees" according to the north-american definition of the term.

So, professional employees in Quebec, and more particularly the engineers, have in fact disregarded the provisions of the Labour Code, and they have fought to develop "syndicalisme de cadres " through incorporation under the Professional Syndicates' Act, direct action and voluntary recognition by the employer, being ready to go on strike eventually to gain recognition by the latter. That is what they did successfully at the Quebec-Hydro in August 1965. The purpose of such an action was to be able to bring into the ranks of the union, not only the mere " employees» covered by the Labour Code, but also all those engineers who may be held to be part of management, so long as they don't have the right to hire, fire or promote other engineers. That is truly the concept of "syndicalisme de cadres ».

We must remember also, that the new Civil Service Act of Quebec (Bill 55) enacted in August 1965 recognizes the right for the employees of the Civil service to organize into unions and to bargain collectively with the government. This new statute considers the government employees as covered generally by the Labour Code, while stipulating certain limitations of its own which, by an large, do not destroy the substance of the rights recognized in the Labour Code.

Members of professional corporations mentioned in the Labour Code, who are " employees » of the Government within the meaning of this Code, may be granted a certification, either for each group of them, or if a majority in the group wishes so, they may join in a more general association including other groups of professionals in order to bargain collectively for them all. They even may join with other groups of professionals, not members of a full corporation (e.g. economists, biologists, etc.) to form a multi-professional association negotiating with the provincial government.

But here again, the big problem appears to be that of delineating the frontier between those professional civil employees who are "employees " under the Code and those who are not. The same attitude as was adopted by the engineers at the Quebec-Hydro, as to "syndicalisme de cadres » is, I think, being adopted by their Civil service employee group and we may suspect that other classes of professionals in the civil service will act along the same pattern. 